\title{
Simulation-Based Sensor Location Model for Arterial Street
}

\author{
Qinxiao Yu, Ning Zhu, Geng Li, and Shoufeng Ma \\ Institute of Systems Engineering, College of Management \& Economics, Tianjin University, Tianjin 300072, China \\ Correspondence should be addressed to Geng Li; ligeng345@126.com
}

Received 25 December 2014; Accepted 26 May 2015

Academic Editor: Essam Radwan

Copyright (C) 2015 Qinxiao Yu et al. This is an open access article distributed under the Creative Commons Attribution License, which permits unrestricted use, distribution, and reproduction in any medium, provided the original work is properly cited.

Traffic sensors serve as an important way to a number of intelligent transportation system applications which rely heavily on realtime data. However, traffic sensors are costly. Therefore, it is necessary to optimize sensor placement to maximize various benefits. Arterial street traffic is highly dynamic and the movement of vehicles is disturbed by signals and irregular vehicle maneuver. It is challenging to estimate the arterial street travel time with limited sensors. In order to solve the problem, the paper presents travel time estimation models that rely on speed data collected by sensor. The relationship between sensor position and vehicle trajectory in single link is investigated. A sensor location model in signalized arterial is proposed to find the optimal sensor placement with the minimum estimation error of arterial travel time. Numerical experiments are conducted in 3 conditions: synchronized traffic signals, green wave traffic signals, and vehicle-actuated signals. The results indicate that the sensors should not be placed in vehicle queuing area. Intersection stop line is an ideal sensor position. There is not any fixed sensor position that can cope with all traffic conditions.

\section{Introduction}

Traffic sensors (e.g., magnetic detectors, cameras, and bluetooth detectors) are widely used in transportation system for systematic surveillance. Various traffic applications need different sensor data. OD estimation may require the traffic counting information on links. Travel time estimation asks for information about link travel time or path travel time. These information can be obtained either from float vehicle such as taxi or cameras which are able to read plate license number. One of the most intuitive pieces of information for advanced traveler information system is travel time. Therefore, a simple and implementation-wise easy method is needed to estimate travel time.

Attempt to estimate arterial street travel time is very challenging. Arterial street traffic is highly dynamic and the movement of vehicles is disturbed by signals and irregular vehicle maneuver. Zhang [1] developed a travel time and journey speed estimation method for freeways by the utilization of volume to capacity ratio, volume, and occupancy. Skabardonis and Geroliminis [2] employed Kinematic wave theory to model the spatial-temporal queueing at the signals. Liu and $\mathrm{Ma}$ [3] proposed that loop detector data and signal phase changes information, which in a high-resolution data context are required to estimate travel time. Without using traditional traffic flow theory, Takaba et al. [4] developed some heuristic models for travel time estimation by using loop detector and license plate reader. A neural network framework is developed to fuse probe vehicle data and loop detector data by Cheu et al. [5]. Dailey and Cathey [6] employed probe sensor to define vehicle speed function. Currently, Li et al. [7] studied that most of the travel time estimation methods or algorithms require many sources of real time data. Investment in these transportation surveillance devices is costly, and thus providing these pieces of real time information is expensive. Among all these traffic sensors, loop detectors are relatively cheap and popular. In addition, loop detectors are also widely used in travel time estimation field $[8,9]$. Due to the high cost of transportation infrastructure, it is necessary to find a way to save investment.

Sensor location problem works for this purpose. It aims to find an optimal sensor placement pattern either in transportation network or freeway. The purpose of sensor placement is mainly for various flow estimations which are OD trips estimation, link flows estimation, path flows estimation, and its related application. Yang and Zhou [10] proposed four sensor location rules in transportation network mainly for OD estimation. This paper can be seen the seminal paper in 
the sensor location literature. Bianco et al. [11] introduced a linear system approach for sensor location problem modeling. Gentili and Mirchandani [12] extended linear system approach by introducing active and passive sensors. Ehlert et al. [13] presented several models to cover as many flows as possible. OD estimation using generalized least-square method is applied to seek for the optimal sensor placement pattern by Fei et al. [14] and Eisenman et al. [15]. In addition to OD estimation, path estimation or identification is another hot topic. Normally, license plate reader is employed to recognize route or estimate route travel time by Castillo et al. [16] and Mínguez et al. [17]. Modeling techniques adopted are integer program. Commercial software or heuristics is used for solving these problems. Hu et al. [18] studied the link sensor placement problem to infer all link flow information of the network of interests. Viti et al. [19] investigated partial observation problems and gave a simple metric for quantify the quality of a sensor placement pattern. Other sensor location problems include mobile sensor routing problem (Zhu et al. [20]), bottleneck identification oriented sensor location problem (Liu and Danczyk [21]), and sensor location problem considering time-spatial correlation (Liu et al. [22]).

Sensor location problem on freeway is relatively limited, particularly for travel time estimation. Kim et al. [23] adopted genetic algorithm to find an optimal sensor placement location on freeway with the minimization of mean absolute relative error. Kianfar and Edara [24] used clustering technology for optimizing freeway traffic sensors. Other freeway sensor location problems employ empirical study methods (Kwon et al. [25]), simulation (Thomas [26]), and dynamic programming (Ban et al. [27]).

Due to the complexity of urban transportation system, none of current studies consider combining travel time estimation method with a sensor location pattern to seek an optimal sensor placement pattern. Another important characteristic of urban transportation system is traffic signal control. This paper attempts to find optimal sensor location pattern with traffic signals. A simulation tool is used to generate basic traffic flow data. The rest of this paper is organized as follows. Section 2 offers a description of travel time estimation method and partition rule in arterial street. Section 3 presents optimal sensor location pattern on a single link. Section 4 gives results for multiple links and also with traffic signal control. Section 5 concludes the whole paper.

\section{Sensor Location Model Description}

2.1. Section Partition Rule for Arterial Street. In our model, a signalized arterial street is partitioned into sections. Each section is associated with a sensor, and the speed of section is represented by the average instantaneous speed (normally, it is collected from the 30s time interval) at the sensor spot. The estimated travel time of each section is calculated by the length and speed of the section. By summing up estimated travel time across all sections, the estimated arterial travel time can be obtained.

In our study, the boundary of section is determined by three sensors, which are located at the section and the adjacent upstream and downstream section, respectively. The total length of signalized arterial is set to $L$, the total number of sensors is $n$, the location of the sensor $i$ is $x_{i}$, and the length of section $i$ is $l_{i}$ which is calculated as follows:

$$
l_{i}= \begin{cases}x_{1}+\frac{x_{2}-x_{1}}{2}, & i=1 \\ \frac{x_{i+1}-x_{i-1}}{2}, & 1<i<n \\ L-x_{n}+\frac{x_{n}-x_{n-1}}{2}, & i=n .\end{cases}
$$

2.2. Travel Time Estimation Model. There are many travel time estimation models, among which the speed-based travel time estimation model is easy-to-operate and widely applied [6-9, 28-30]. These three models proposed by Li et al. [7] are adopted in our study. The principle of all these three models is to calculate travel time according to the spot-speed obtained by sensors.

The first model is instantaneous model. A vehicle is supposed to enter the arterial street at time $k$. The detected speed at time $k$ is considered as the average speed of vehicles at that section. The travel time of vehicle at section $i$ is denoted as $t(i, k)$ :

$$
t(i, k)=\frac{l_{i}}{v(i, k)},
$$

where $l_{i}$ is the length of the section $i$ and $v(i, k)$ is the measured speed of section $i$ at time $k$. The travel time $T(k)$ of vehicle passing through the entire signalized arterial street is the sum of all sections' travel times:

$$
T(k)=\sum_{i=1}^{n} t(i, k) .
$$

In the instantaneous model, speeds from only one point on each section are used to estimate travel time, while ignoring the speed variations within a section. This does not meet the authentic traveling situation of vehicles. However, from the perspective of calculation, when the travel time is fixed, its corresponding average speed is fixed. Therefore, the sensor location pattern is very critical. A suitable senor location pattern can accurately capture the average travel time of its spatial influence area. On the other hand, all speeds are collected at the time of vehicle entering the arterial. The speed associated with the downstream section will not change dramatically when the vehicle traverses the arterial. These two reasons result in travel time estimation error. The other two speed estimation models are Time Slice Model and Dynamic Time Slice Model.

The difference among the three travel time estimation models lies in the calculation method for vehicle speed on each section, which is the main factor that affects error. These three models all use the speed measured by sensors, so the travel time estimation error is caused by not only inevitable calculation error, but also the error arising from the location of sensor in arterial street. Different combinations of sensor locations generate different section partitions, thus, resulting in different estimation errors. 


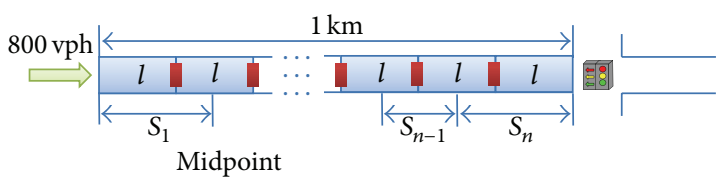

FIGURE 1: Partitioned arterial street with traffic signal.

To compare the differences among these three models, numerical experiments are conducted for the identical sensor location pattern. In our study, a traffic simulation tool is used for a street with length $1 \mathrm{~km}$ as shown in Figure 1. Intersection signal cycle is $100 \mathrm{~s}$, green light duration is $60 \mathrm{~s}$, and the vehicle enters the street with a speed of $3.6 \mathrm{~km} / \mathrm{h}$. The maximum speed is $57.6 \mathrm{~km} / \mathrm{h}$. The vehicle's arrival rate is $800 \mathrm{vph}$ and obeys Poisson distribution. Sensors are evenly distributed on the road at equal interval of $l$. The influential area of each sensor is shown in Figure 1. The number of sensors is increased from 1 to 10 . Travel time is estimated with above-mentioned three methods, respectively.

In order to compare the resulted obtained by these three methods, we use three measures for evaluation which are the mean absolute error (MAE), root mean square error (RMSE), and mean absolute relative error (MARE), respectively.

$$
\begin{aligned}
\text { MAE } & =\frac{1}{n} \sum_{i=1}^{n}\left|\mathrm{ett}_{i}-\mathrm{gttt}_{i}\right|, \\
\mathrm{RMSE} & =\sqrt{\frac{1}{n} \sum_{i=1}^{n}\left(\mathrm{ett}_{i}-\mathrm{gttt}_{i}\right)^{2},} \\
\text { MARE } & =\frac{1}{n} \sum_{i=1}^{n} \frac{\left|\mathrm{ett}_{i}-\mathrm{gttt}_{i}\right|}{\mathrm{gttt}_{i}} .
\end{aligned}
$$

ett $_{i}$ refers to the estimated travel time, gttt ${ }_{i}$ refers to the ground-truth travel time, and $n$ refers to the number of vehicles. Through 10 traffic simulations, 10 groups of travel time $\left\{S_{r} \mid S_{r}=\left(\mathrm{gttt}_{r 1}, \mathrm{gttt}_{r 2}, \ldots, \mathrm{gttt}_{r n}\right), r=1,2, \ldots, 10\right\}$ are obtained. These three travel time estimation methods were used to calculate the corresponding estimated travel time and the $\mathrm{MAE}_{r}, \mathrm{MARE}_{r}$, and $\mathrm{RMSE}_{r}$ respectively. Finally, 10 sets of three measures are obtained for each travel time estimation method. The mean values and standard deviations are calculated according to these 10 sets of data. The experimental results are shown in Figure 2.

As shown in Figure 2, the three estimation models have little differences in most cases. Particularly, the time slice model and dynamic time slice model almost obtain the same results. The instantaneous model outperforms other two estimation models, although instantaneous model (IM) only makes estimation according to the traffic condition when vehicle enters the street. But under the traffic signal control, the traffic flow has certain reproducibility. The vehicle's travel time can be accurately estimated when the vehicles traverse the arterial street smoothly. Therefore, in subsequent experiments, IM method is adopted to estimate the travel time.

\section{Sensor Location Setting in Single Link}

In the urban transportation network, the movement of vehicles is with some regularity due to the traffic signal control. Generally it can be summarized as, after passing through previous intersection, vehicles enter the street at low speed or original speed. Then, the vehicles accelerate to the maximum allowable speed and keep moving. When approaching the next intersection, it determines whether to slow down or keep moving at the original speed according to traffic signals. The speed contour profile is shown in Figure 3.

The key point of estimating vehicle's travel time on a certain link is to find the vehicle's average speed on that link. Vehicle moves with different speed at different positions of the link. In order to accurately estimate travel time, we need to find an appropriate location for the sensor, making sure that the instantaneous speed is close to the average speed. Therefore, the location of sensor has great impact on the travel time estimation error. Figure 4 shows the relationship between travel time estimation error (MARE) and different sensor locations when a sensor is placed on a $1 \mathrm{~km}$ link under different arrival rates.

As can be seen from Figure 4, the smallest travel time estimation error is obtained when the sensor is placed at the $50 \mathrm{~m}$, and the mean travel time estimation error of the four arrival rates is about $8 \%$. In the link from $100 \mathrm{~m}$ to $850 \mathrm{~m}$, the sensor location has little impact on travel time estimation and the error is about $29 \%$. In the $100 \mathrm{~m}$ of downstream section, the travel time estimation error increases about $120 \%$.

Sensor location pattern is closely related to the vehicle's trajectory on the link. (i) If a vehicle enters the intersection at a slow speed and the sensor is placed at the inlet $(0 \mathrm{~m})$ position, where the average speed is small, the estimation error will be large. (ii) If the vehicle accelerates to the maximum speed after entering the link, the vehicle speed varies greatly within this distance (from 3.6 to $56.6 \mathrm{~km} / \mathrm{h}$ ), so it is easy to find a position that can represent the average speed of the link. (iii) The vehicle runs at the limited speed in the middle section of the link, and the speed fluctuation is small. Therefore, no matter where the sensor is placed, the detected speed is almost equal to the maximum speed. Thus, it cannot reflect the average speed on the link. (iv) Within the $100 \mathrm{~m}$ in the downstream, vehicles enter the queuing area. The length of queue increases as the arrival rate increases. When the sensor is closer to the intersection, the sensor is likely to be occupied by vehicles. When the sensor is occupied, it cannot detect speed. The estimation error is large. However, when the arrival rate is less than the saturation volume, the number of queuing vehicles is small. All the vehicles can pass through the intersection in a signal cycle. The proportion of sensor's occupied time is small. Therefore, the travel time can be estimated. When the arrival rate is greater than the saturated volume, vehicles at the tail of the queue need to wait for two or more signal cycles before passing through the intersection. The sensor is more likely to be occupied and cannot obtain vehicle speed during a long period of time. 

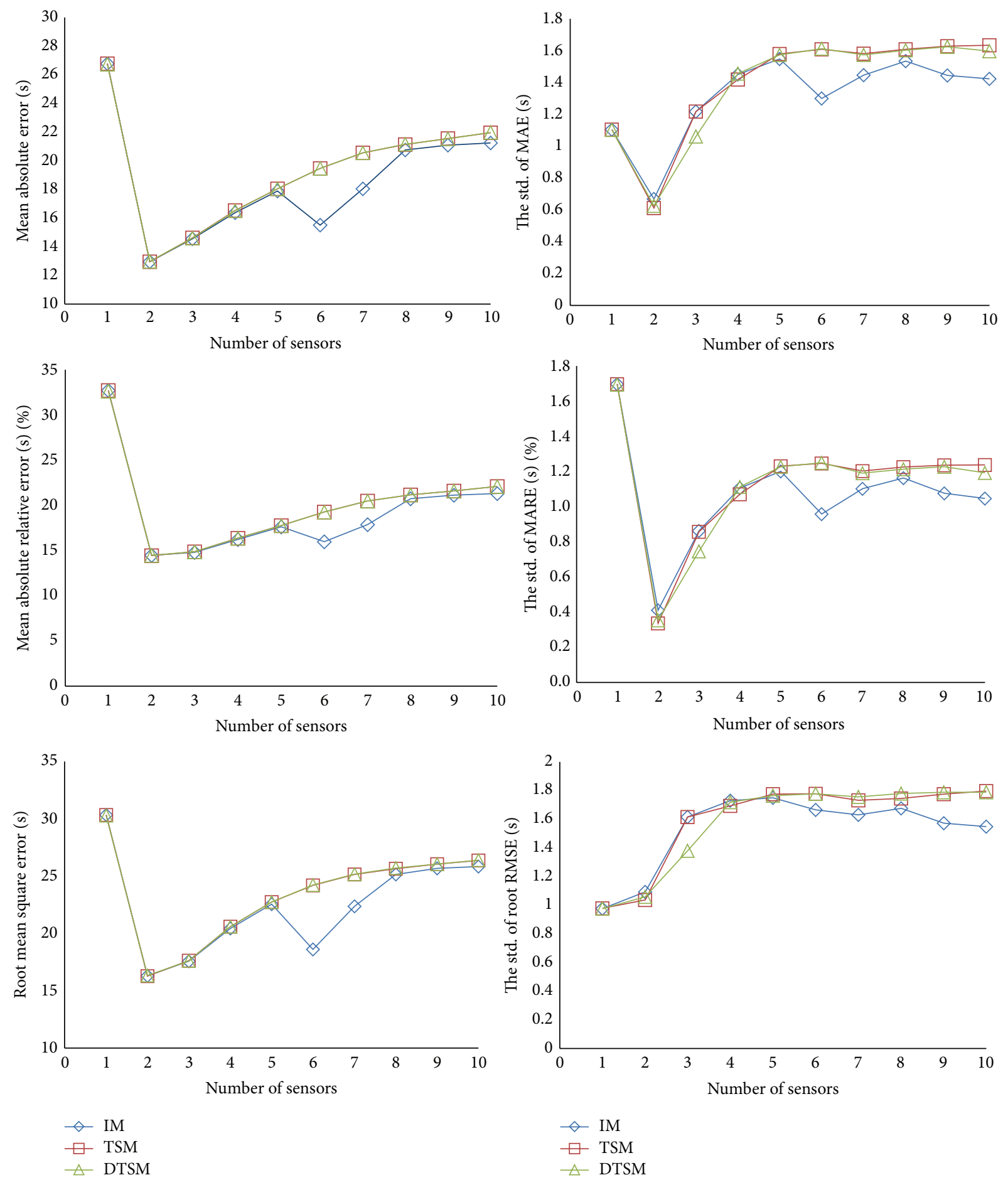

Figure 2: Errors and their standard deviations versus number of sensors.

\section{Sensor Location in Arterial Street}

4.1. Sensor Location Model Description. A signalized arterial street is usually composed of many links, and there is a traffic signal between every two links. In order to study sensor placement pattern in such a signalized arterial street, we divide arterial street into equal-length cells, as shown in
Figure 5. If a cell is equipped with a sensor, then the sensor will be placed on the cell's right boundary. Section is defined as the influential area of the sensor. The partition method is given in previous section.

Assume that the entire horizon of the study is $T$ and a total of $M$ vehicles pass through the entire street. $K$ sensors will be placed on the arterial street; that is, there will be $K$ sections. 


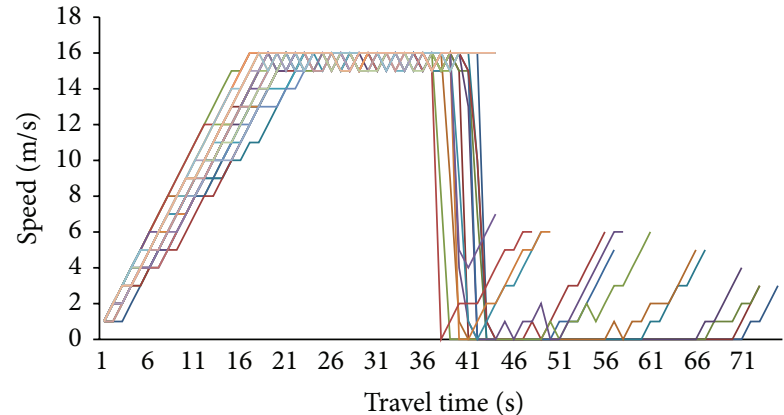

FIgURE 3: Speed contour profile in a signal cycle.

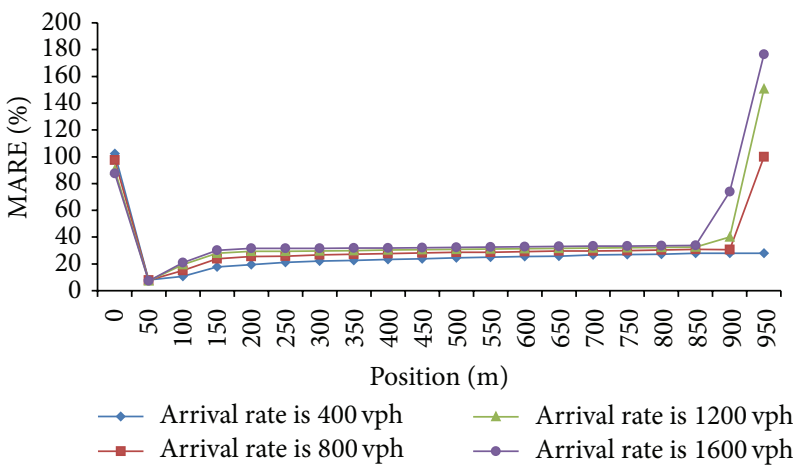

FIGURE 4: Estimate error versus different sensor locations.

The actual travel time of the $m$ th vehicle that passes through the arterial is GTTT $_{m}$, which is obtained by simulation. The estimated travel time is $\mathrm{ETT}_{m}$, which is the total sum of $K$ sections' estimated travel time $\mathrm{ETT}_{m k}$. The goal of sensor location model is to minimize the error between estimated travel time and actual travel time. The decision variable of the model is $x_{i} \in\{0,1\}$, which indicates whether the sensor is placed on $i$ th cell. The model is shown as follows and is called M1:

M1:

$$
\begin{aligned}
\min & \frac{1}{M} \sum_{m=1}^{M}\left|\frac{\left(\sum_{k=1}^{K} \mathrm{ETT}_{m k}\right)-\mathrm{GTTT}_{m}}{\mathrm{GTTT}_{m}}\right|, \\
\text { subject to: } & \sum_{i=1}^{N} x_{i}=K, \\
& x_{i} \in\{0,1\}, \\
& Y \text { is set of the index of } x_{i} \\
& \text { where } x_{i}=1,
\end{aligned}
$$

$y_{k}$ is the $k$ th element in $Y$,

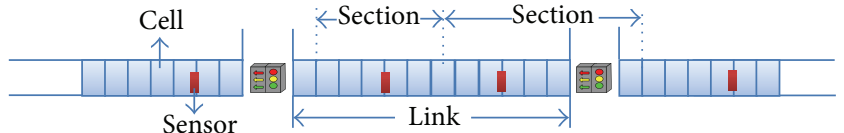

FIgURE 5: Partitioned arterial street.

$s_{k}$

$$
= \begin{cases}\frac{y_{k+1}-y_{k-1}}{2}, & k=2, \ldots, K-1 \\ y_{1}+\frac{y_{2}-y_{1}}{2}, & k=1 \\ y_{K}+\frac{N-y_{K}}{2}, & k=K,\end{cases}
$$

$$
\mathrm{ETT}_{k}=\frac{s_{k}}{v_{k}} \cdot l \text {. }
$$

In M1, $M$ is the number of total vehicles, $N$ is the number of cells, and $K$ is the number of sensors, namely, the budget constraint. $x_{i}$ is the decision variable, and $x_{i}=1$ indicates that the $i$ th cell has a sensor; otherwise, $x_{i}=0$. $y$ represents the set of cells that are installed with sensors, wherein the total number of elements is $K$. For instance, if the entire arterial street is divided into 10 cells, budget constraints are three sensors, $Y=\{3,6,8\}$ means that sensors are placed in the 3th, 6th, and 8th cells. $y_{k}$ refers to the $k$ th element of set $Y$, and $S_{k}$ is the coverage area of $k$ th sensor, namely, the number of cells contained in the $k$ th section, which is determined by the position of adjacent upstream and downstream sensors. When the positions of a group of sensors are given, the length of $K$ sections can be calculated. Also taking $Y=\{3,6,8\}$ as an example, $s_{1}$ covers 4.5 cells, $s_{2}$ covers 2.5 cells, and $s_{3}$ covers 3 cells. $V_{k}$ is the average speed of section $k$ which is calculated according to the speed detected by corresponding sensor. After solving this model, the optimal sensor placement pattern can be obtained. Due to the complexity of the combinational optimization problem, exact algorithm is very hard. Thus, in our study, genetic algorithm is employed.

In this section, a mathematic model is proposed to solve the sensor placement problem in arterial street. It is a $0-1$ programming model. The objective function is to minimize the relative travel time estimation error between estimated and actual travel time. The input data of the model is travel time information of all vehicles in computer simulation which is treated as ground-truth travel time. Once the groundtruth travel time information is given, and our proposed mathematical programming model can decide the optimal sensor placement pattern that minimizes the relative travel time estimation error. Therefore, our model is a mathematical model. In addition, the model is deterministic.

4.2. Case Study. In this study, we only consider the influence of vehicle arrival rate and traffic signal strategy on sensor placement pattern which are two major factors that affect travel time on urban network. In the simulation, the signalized arterial street is set as $3 \mathrm{~km}$ long, and it is composed of 6 links. Each link is $0.5 \mathrm{~km}$ long. Each link is divided into 


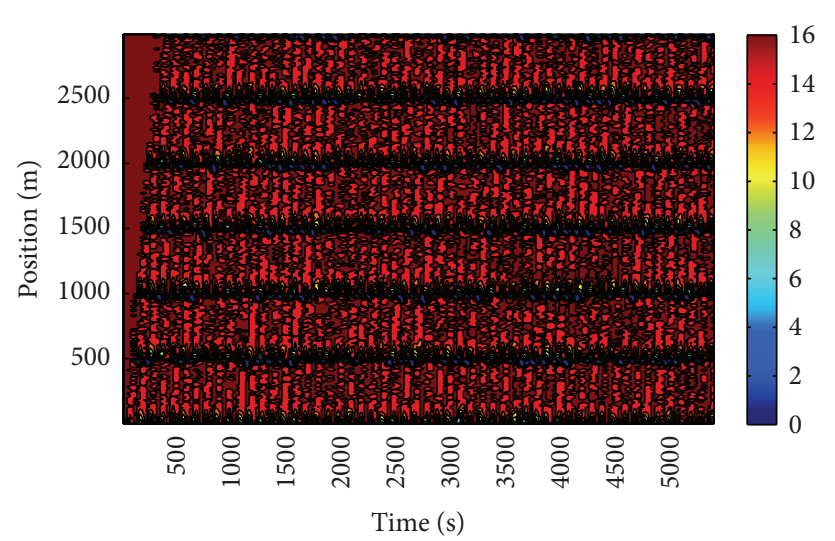

(a) Arrival rate is $400 \mathrm{vph}$

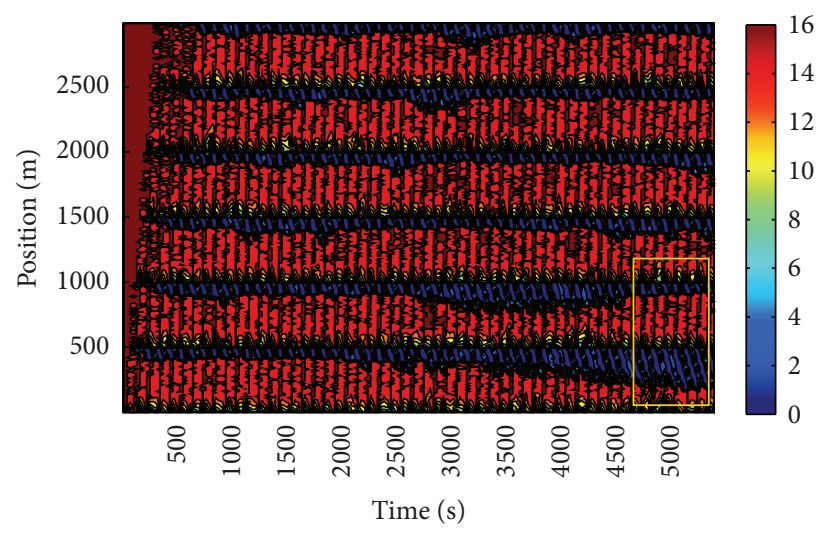

(c) Arrival rate is $1200 \mathrm{vph}$

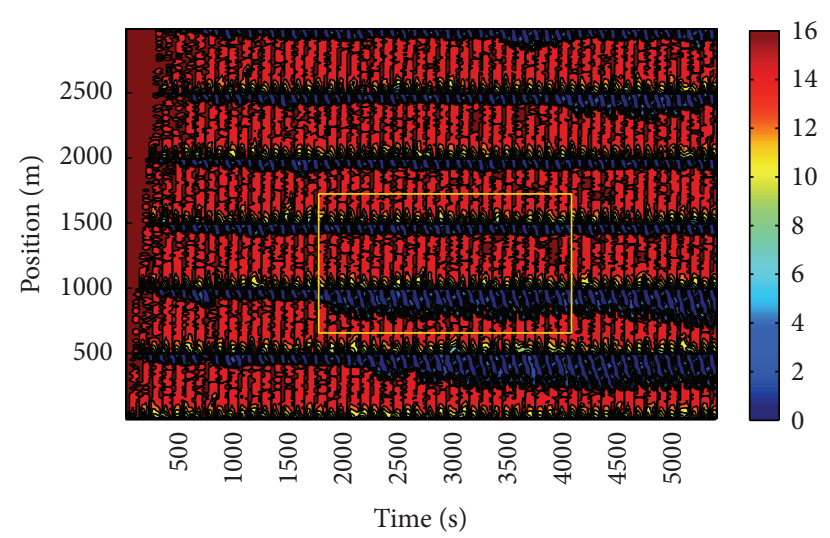

(b) Arrival rate is $800 \mathrm{vph}$

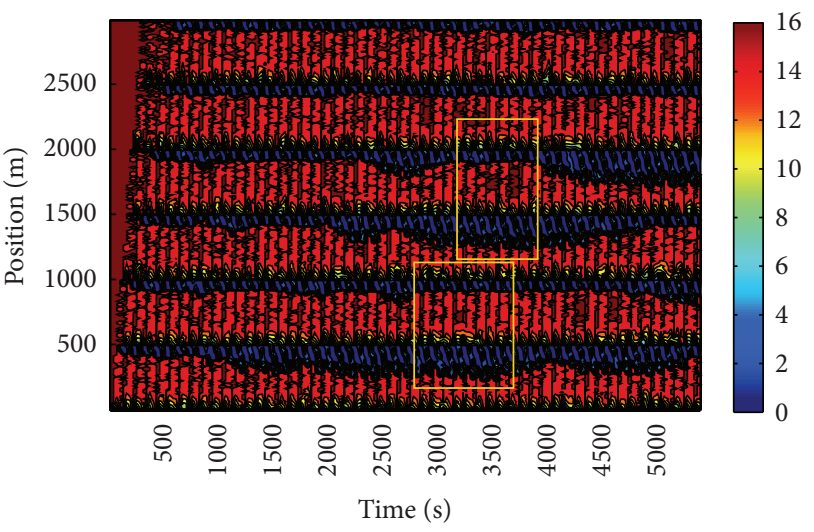

(d) Arrival rate is $1600 \mathrm{vph}$

FIGURE 6: Speed contour plot in synchronized traffic signals.

five cells, and each cell is $0.1 \mathrm{~km}$ long. The limited speed is $57.6 \mathrm{~km} / \mathrm{h}$. Vehicle arrival rate at the entrance is set as $400 \mathrm{vph}, 800 \mathrm{vph}, 1200 \mathrm{vph}$, and $1600 \mathrm{vph}$, respectively, and arrival rate obeys Poisson distribution. When the arrival rate exceeds the intersection capacity, there will be congestion. In each simulation, the arrival rate is fixed. In the urban transportation network, traffic signals strategy can greatly affect the capacity of each intersection and thereby affect the trajectory of vehicle after entering the arterial street. Therefore, we need to consider different traffic signals strategies. In this study, we consider three common traffic signals strategies: synchronized traffic signals, green wave traffic signals, and vehicle-actuated signals. In the experiment, we analyzed three strategies separately under different vehicle arrival rates.

4.2.1. Synchronized Traffic Signals. Synchronized control refers that all intersections of the arterial street use the same signal configuration and display the same traffic signal at the same time. In the simulation, the signal cycle is set as $100 \mathrm{~s}$, and green ratio is $50 \%$. Vehicle's speed trajectories under different arrival rates are shown in Figure 6. The $x$ axis represents time, and the $y$-axis represents the vehicle position. Speed is represented by different colors. Color change from red to blue refers to the gradual decrease of speed. Each row represents the speed variation of the same position at different time points. Each column represents the speed of each position at the same time.

According to Figure 6(a), the arrival rate is $400 \mathrm{vph}$, which is far less than the intersection capacity. In the whole simulation horizon, the number of vehicles that arrive at the intersection is small, and all vehicles can pass through the intersection within one signal cycle, so the queue length of each intersection is short. In Figure 6(b), the vehicle arrival rate is approximately equal to the intersection capacity. Because the arrival rate obeys Poisson distribution, vehicles have different actual arrival rates in each signal cycle. Some arrival rates are greater than the intersection capacity, and some are less than the intersection capacity. When the arrival rate is greater than the intersection capacity in a signal cycle, there will be multiple queues. While the queuing at the downstream intersection spreads to the upstream, it reduces the actual capacity of upstream intersection, making the queuing at upstream section much longer as the first and second intersections after $2000 \mathrm{~s}$ shown in Figure 6(b). When the arrival rate is much greater than the intersection capacity, some queuing vehicles may not be able to pass through the intersection within one signal cycle. Vehicle release rate will be exactly equal to the intersection capacity; that is, the arrival rate of the next intersection is equal to the intersection 
TABLE 1: Comparison among different arrival rates.

\begin{tabular}{lcc}
\hline $\begin{array}{l}\text { Arrival rate } \\
\text { (vph) }\end{array}$ & $\begin{array}{c}\text { Number of } \\
\text { sensors }\end{array}$ & Sensor location pattern \\
\hline 400 & & $500,600,1100,1700,2900,3000$ \\
800 & 6 & $500,600,2000,2300,2500,3000$ \\
1200 & & $1000,1100,1300,2100,2500,2900^{*}$ \\
1600 & & $1100,1200,1300,800,1600,2500,2600$ \\
\hline 400 & 5 & $200,600,1100,1800,2200$ \\
800 & & $1000,1200,2000,2300,2900^{*}$ \\
1200 & & $100,600,1600,2200,2800$ \\
1600 & & $500,800,1900,2300$ \\
400 & 4 & $200,500,1100,2800,2700,3000$ \\
800 & & $200,400^{*}, 600,2900$ \\
1200 & & \\
1600 & &
\end{tabular}

${ }^{*}$ The sensor is located in the queuing area.

capacity. This intersection will not form a secondary queue. As shown in the yellow rectangle of Figures 6(b), 6(c), and $6(d)$, if the queue of vehicles at the upstream intersection is too long, then the queue of vehicles at the downstream intersection is short.

Table 1 shows the optimal sensor placement pattern of certain number of sensors at different arrival rates. Some observations can be summarized as follows; (i) among the 12 optimal sensor placement patterns, only three patterns set the sensor in the queuing area (blue area in Figure 6). The bold numbers in Table 1 represent the queuing area, and the corresponding influence areas of the 3 sensors are very short which are $300 \mathrm{~m}, 400 \mathrm{~m}$, and $200 \mathrm{~m}$, respectively. In these three areas, blue area takes up a large proportion; that is, the average speed of vehicles is small. The queuing is fairly severe. Thus, when sensor is placed in the queuing area, the corresponding influence area is with long queuing length. This is because the average speed in queuing area is small. It has great impact on the estimated travel time. The sensor should not be placed in the queuing area. When sensor is placed in the queuing area, its corresponding detection range should not be too large, and it should be able to accurately capture the length of the queuing. (ii) Among the 12 optimal placement patterns, 9 patterns set sensors at one or more stop line positions (positions 500, 1000, 1500, 2000, 2500, and 3000). Compared with other locations, the average speed of vehicles at stop lines has the largest variations, and the speed profile at stop line is repetitive with regard to cycle time. When the red traffic light is on, the speed is 0 . When the green light is turned on, the speed gradually increases to the maximum speed of $57.6 \mathrm{~km} / \mathrm{h}$. When the arrival rate is small, the number of queuing vehicles is small, and then the average speed is high. When the arrival rate is large, the number of queuing vehicles is large, and then the average speed is low. In addition, speed variation is determined by both the upstream queuing and downstream traffic congestion situation. Thus, when there are a lot of vehicles queuing at both the upstream and downstream links, the sensor at stop line can timely and flexibly detect the variation of traffic situation. (iii) When the number of sensors is fixed, the corresponding sensor

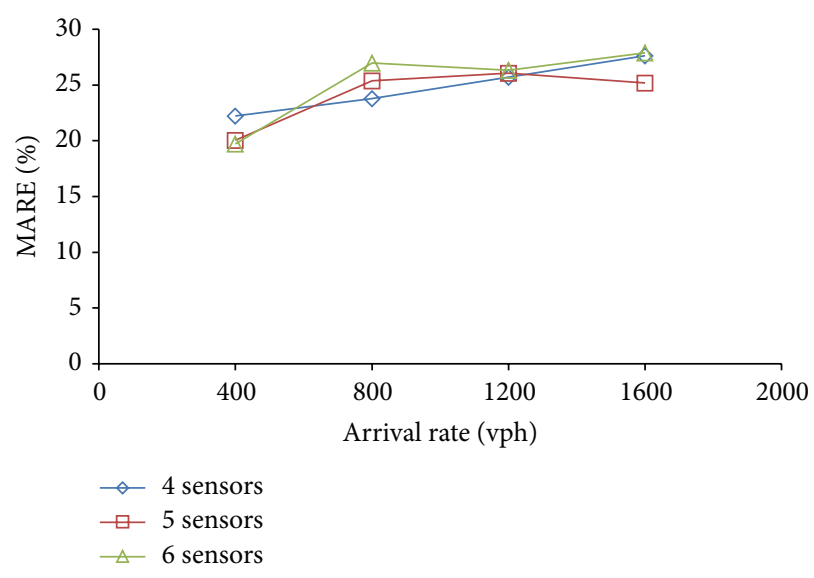

FIGURE 7: Comparison among MARE in different number of sensors for synchronized traffic signals.

placement patterns of different arrival rates vary greatly. This is because different arrival rates leads to different queue lengths, so the position of sensor should be determined by the road conditions.

In Figure 7, each point represents the travel time estimation error under the optimal sensor placement pattern. This pattern is obtained under certain arrival rate and certain number of sensors. When the arrival rate is $800 \mathrm{vph}, 4$ sensors are better than 5 or 6 sensors. When the arrival rate is $1600 \mathrm{vph}, 5$ sensors are better than 6 sensors. Therefore, optimizing the sensor placement pattern can reduce errors and number of sensors. When the number of sensors is 4, MARE is in proportional with the arrival rate; that is in this case, the estimation error cannot be reduced by changing sensor location. However, the estimation error can be reduced by increasing the number of sensors.

4.2.2. Influence of Green Ratio. Green ratio is an important parameter in traffic signals strategy. Congestion occurs when traffic volume is greater than the intersection capacity. In order to timely dissipate queuing vehicles, green ratio should be lengthened. When traffic volume is less than the intersection capacity, the green ratio should be carefully reduced. Green ratio will directly affect intersection capacity. When the arrival rate is a constant, vehicle queuing will change, thus affecting the position of sensor.

In order to study the influence of sensor location on estimated travel time under different green ratios, we set three green ratios, which are $40 \%, 50 \%$, and $60 \%$, respectively. Other parameters are the same as previous section. By using genetic algorithm, the optimal sensor placement pattern and the travel time estimation errors are obtained. As shown in Figure 8, each point represents the travel time estimation error of the optimal sensor placement pattern. It can be seen from Figure 8 that, regardless of the number of sensors, small green ratio causes larger travel time estimation error. The reason could be explained as that traffic condition becomes 

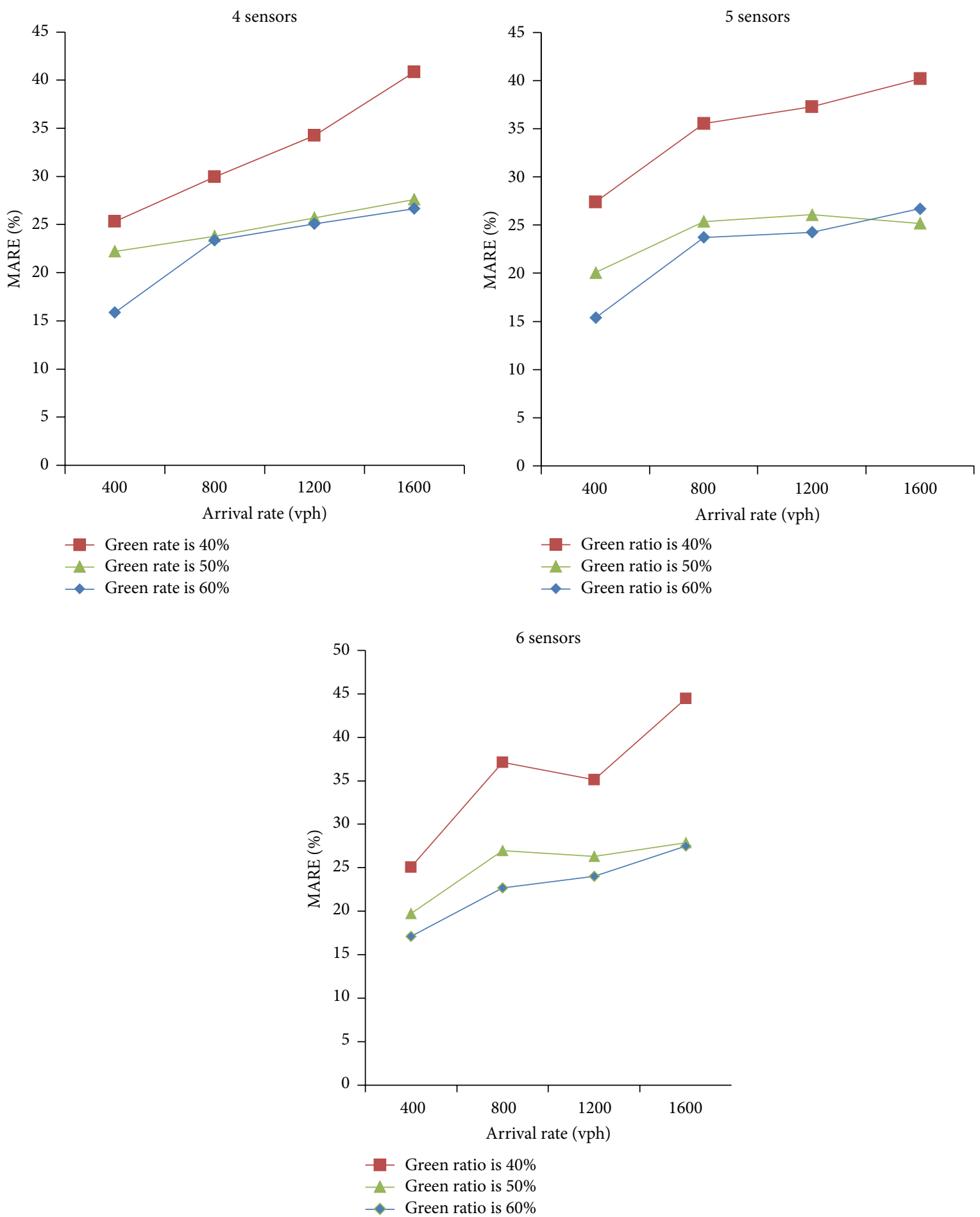

Figure 8: Comparison among different green ratios.

congested and complicated as the green ratio reduced. Therefore, it is hard to predict the travel time.

4.2.3. Green Wave Traffic Signals. Green wave control means that if a vehicle passes through one intersection at a given speed with a green light phase, it will pass through all the downstream intersections at green light phase [31]. The offset time of adjacent intersection's traffic signal equals to the length of the section divided by the given speed. If the vehicle meets a red light phase at the intersection, it will stop and wait. When the light turns green, the vehicle accelerates from zero to the limited speed and moves to the next intersection. The average speed of vehicle on the link is slightly less than the 
TABLE 2: Comparison among different arrival rates.

\begin{tabular}{lcc}
\hline $\begin{array}{l}\text { Arrival rate } \\
\text { (vph) }\end{array}$ & $\begin{array}{c}\text { Number of } \\
\text { sensors }\end{array}$ & Sensor location pattern \\
\hline 400 & & $600,700,900,1500,2600,3000$ \\
800 & 6 & $700,1000,1300,1400,2300,2700$ \\
1200 & & $1500,1700,1800,2100,2600,2800$ \\
1600 & & $800,1200,1400,2300,2400,2800$ \\
\hline 400 & & $1400,1900,2200,2500,3000$ \\
800 & 5 & $900,1300,2700,2800,3000$ \\
1200 & & $900,1300,1400,2500,2900$ \\
1600 & & $1200,1500,1600,2400,3000$ \\
\hline 400 & 4 & $1200,1500,1100,1300,2800,2800$ \\
800 & & $1300,1500,2600,3000$ \\
1200 & &
\end{tabular}

limited speed and travel time is slightly larger than the offset time. Therefore, during the green light period, if the number of queuing vehicles exceeds the intersection capacity, vehicles in the front of queue can successfully pass through the next intersection. However, vehicles in the tail part of the queue will meet red light at the next intersection.

In our simulation, the signal cycle is set as $100 \mathrm{~s}$. Green ratio is $50 \%$. Each link is $0.5 \mathrm{~km}$ long. The limited vehicle speed is $57.6 \mathrm{~km} / \mathrm{h}$. The relative offset time between adjacent intersections is $32 \mathrm{~s}$. Therefore, there will be a band along with the arterial street. As long as the vehicle arrives within the band and keeps moving at the limited speed of $57.6 \mathrm{~km} / \mathrm{h}$, it can travel smoothly through the all intersections. Figure 11 shows the speed trajectories under four different arrival rates. Generally speaking, vehicles can move at the maximum speed on the road most of the time and can smoothly pass through each intersection. If the arrival rate is greater than the intersection capacity, the first intersection will have a small number of queuing vehicles; this is, because some vehicles enter the arterial street at a smaller speed, their travel time on the link is greater than the travel time of vehicle which moves at the limited speed (offset time). They will meet red light at the first intersection.

Table 2 shows the corresponding optimal sensor placement patterns of fixed number of sensors for different arrival rates. All sensors are set after the first intersection. This is because the first link has stable traffic condition without any fluctuations under different arrival rates. This can be seen from the four subgraphs of Figure 9. In the section from $0 \mathrm{~m}$ to $500 \mathrm{~m}$, the vehicle accelerates to maximum speed and moves to the first intersection after entering the arterial. In this section, the speed trajectory is very similar. Besides, like the synchronized traffic signal strategy, 10 out of the 12 patterns set the sensor on one or more stop lines.

Figure 10 shows the estimation error under the optimal sensor placement pattern for green wave traffic signal control. Compared with other two signals strategies, it is easier to estimate the travel time under green wave control. The estimation error is less than $2 \%$. Regardless of the arrival rate, the travel time estimation error is minimized when the number of detectors is 4 . This is because that the traffic condition is simple under the green wave traffic signals. When the traffic is not complicated, less sensors should be placed. When traffic condition is very complicated, more sensors should be placed.

4.2.4. Vehicle-Actuated Traffic Signals. The above two traffic signals strategies are fixed traffic signal control method. They are developed based on historical data. The disadvantage of these strategies is that it is unable to meet real-time changes of traffic flow. In order to overcome this deficiency, vehicleactuated signals [32] are adopted. This strategy changes the green time adaptively according to real-time traffic volume.

In the simulation of vehicle-actuated control, we set each intersection's signal cycle as $100 \mathrm{~s}$. The minimum green time is $50 \mathrm{~s}$. The maximum green time is $70 \mathrm{~s}$. The unit extension interval is $3 \mathrm{~s}$. When the arterial street gets the access right, the signal system will first give the signal phase a minimum green time of $50 \mathrm{~s}$, enabling the vehicle that has arrived at the intersection to pass through the intersection. If there is no vehicle after this, the access right will be transferred to the subsequent link. If a vehicle is detected within the green time, the green time will be extended a unit time interval of $3 \mathrm{~s}$. The maximum extension can be $70 \mathrm{~s}$.

Figure 11 is the speed trajectory for 4 different arrival rates. Compared with the synchronized traffic signals control, the queuing length at the intersection is shorter, and largescale long queue appears only once when the arrival rate is $1600 \mathrm{vph}$. When the traffic volume is large, the signal system can detect the changes of volume, increasing the green time in a timely manner. As a result, intersection capacity is improved and vehicle queue length is reduced. By comparing Figures 6 and 11 , it can be seen that, under the vehicle traffic signals control, the average vehicle queue length is short.

The optimal sensor location pattern under vehicleactuated traffic signals control is shown in Table 3. The corresponding travel time estimation error of each optimal sensor mode is shown in Figure 12. Similar to synchronized traffic signals control, among the 12 optimum placement patterns, only three patterns set the sensor in the queuing area (see the bold numbers in the table). Two of these three sensors have small detection coverage area, which are $250 \mathrm{~m}$ and $400 \mathrm{~m}$, respectively. 12 sensor location patterns all choose to place the sensor on a stop line. As can be seen from Figures 11(c) and 11(d), blue area takes up a large proportion in these two areas; that is, the average speed of vehicles is small and the queuing situation is severe. Thus, when the sensor is located in queuing area, its corresponding influence area is the area with long queues. This is because the average speed of queuing area is small which has great impact on the estimation of travel time. The sensors should be avoided from being placed in queuing area. When the sensor is placed in the queue area, its corresponding influence area should not be too large. It should accurately correspond to the length of the queue. In addition, it can be seen from Figure 12 that (i) the travel time estimation error calculated by different number of sensors differs slightly for different arrival rates. (ii) As for a fixed number of sensors, travel time estimation error increases as the arrival rate increases. (iii) Regardless of 


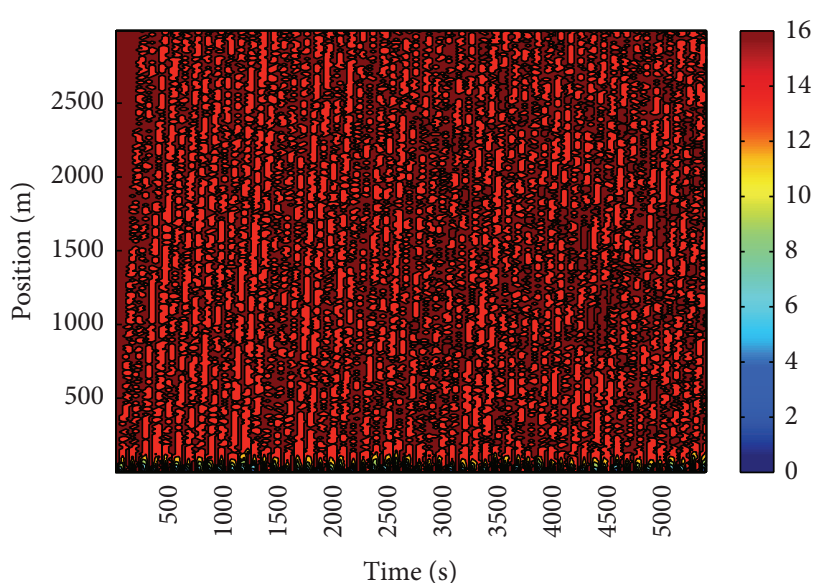

(a) Arrival rate is $400 \mathrm{vph}$

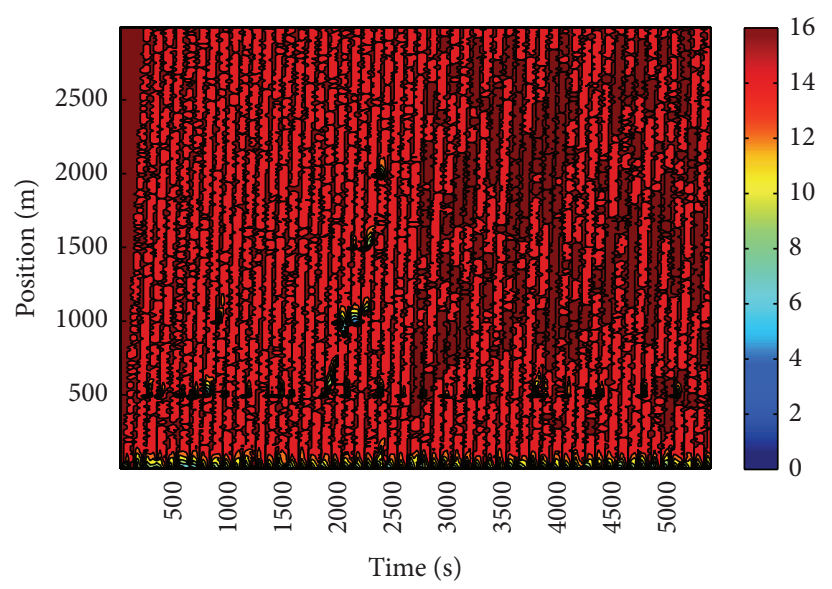

(c) Arrival rate is $1200 \mathrm{vph}$

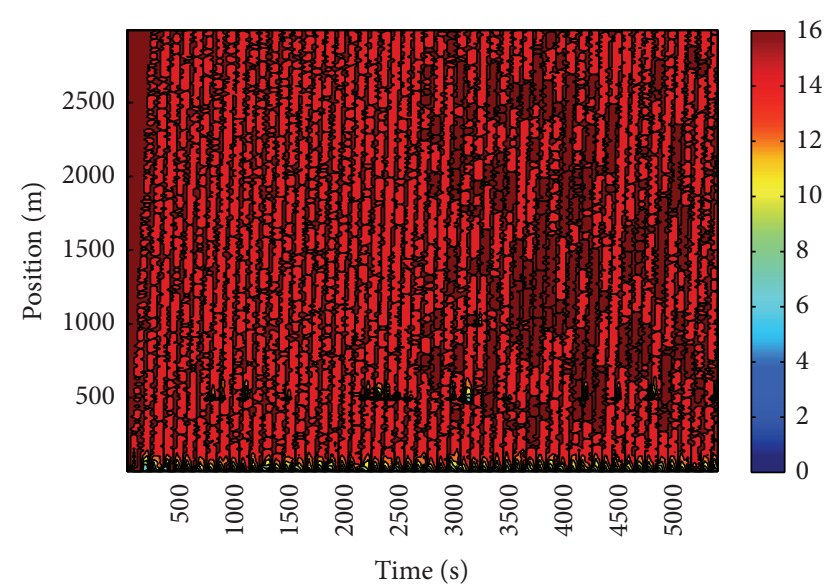

(b) Arrival rate is $800 \mathrm{vph}$

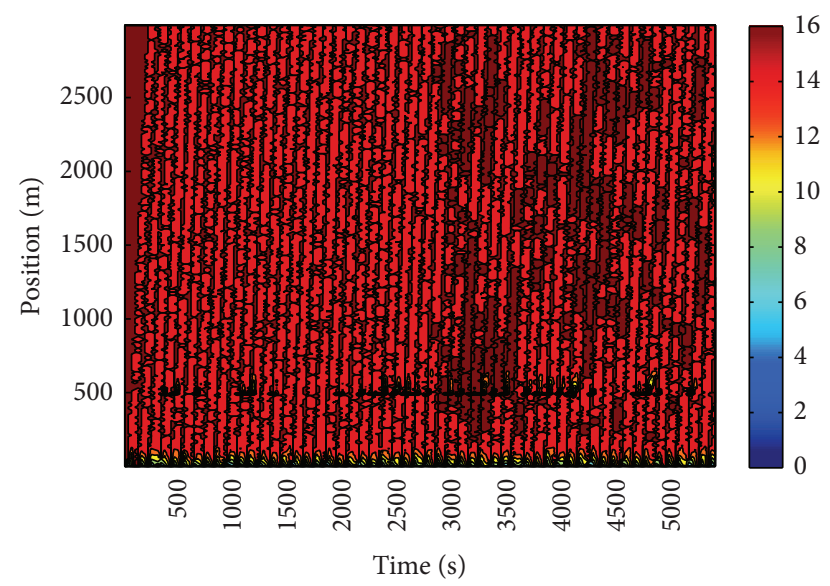

(d) Arrival rate is $1600 \mathrm{vph}$

FIGURE 9: Speed contour plot in green wave traffic signals.

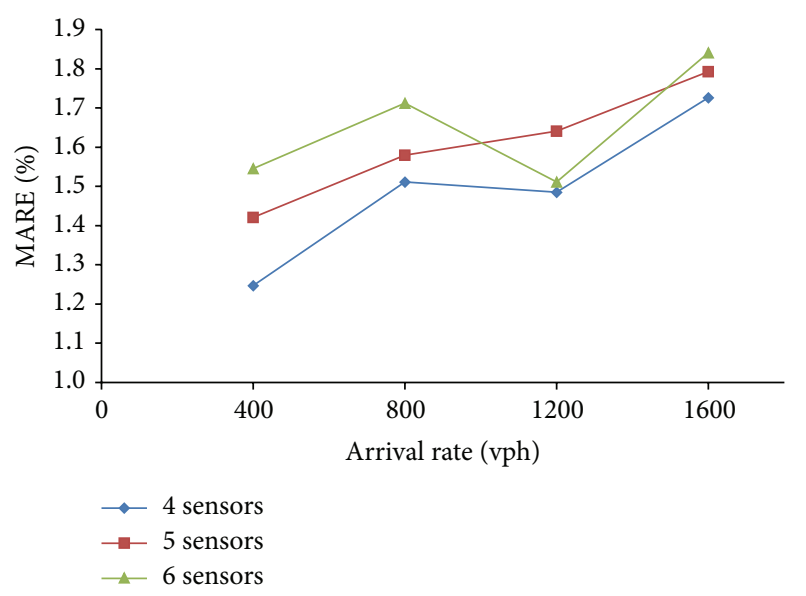

FIgure 10: Comparison among MARE in different number of sensors for green wave traffic signals.

arrival rate, the estimated travel time predicted by 6 sensors is the most accurate. The number of arrived vehicles within one signal cycle changes the green time length adaptively, resulting in the change of intersection capacity. The traffic condition of each link becomes more dynamic and random which differs greatly from the cyclical and repetitive traffic condition under synchronized control. Therefore, it requires more sensors to estimate travel time.

Through experimental analyses of these three traffic signals strategies, observations are stated as follows: (i) when the sensor is located in the queuing area, its corresponding influence area is short. Its length should be close to the queuing length. Therefore, when a sensor is located in the queuing area, some sensors should be set in the adjacent upstream and downstream areas in a cooperative manner. (ii) Stop line is an ideal sensor position place. Compared with other location places, the average speed of vehicles at stop lines has the largest variations. Small arrival rate may cause fewer queuing vehicles. The average speed is high. A large arrival rate may cause more queuing vehicles, where the average speed is low. The sensor can timely and flexibly reflect the traffic conditions. (iii) Under simple traffic situation where vehicle speed is stable with small speed fluctuation, few sensors can accurately estimate travel time. Otherwise, more sensors are needed. 


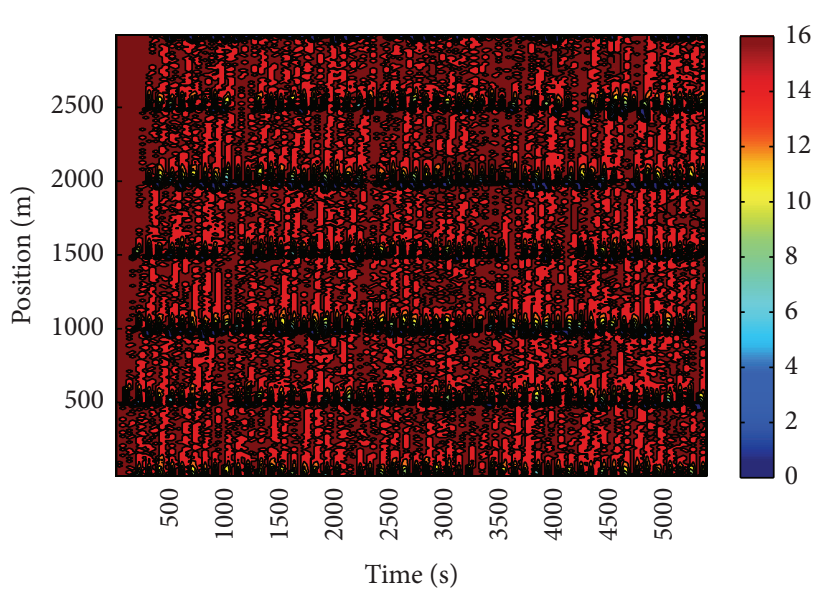

(a) Arrival rate is $400 \mathrm{vph}$

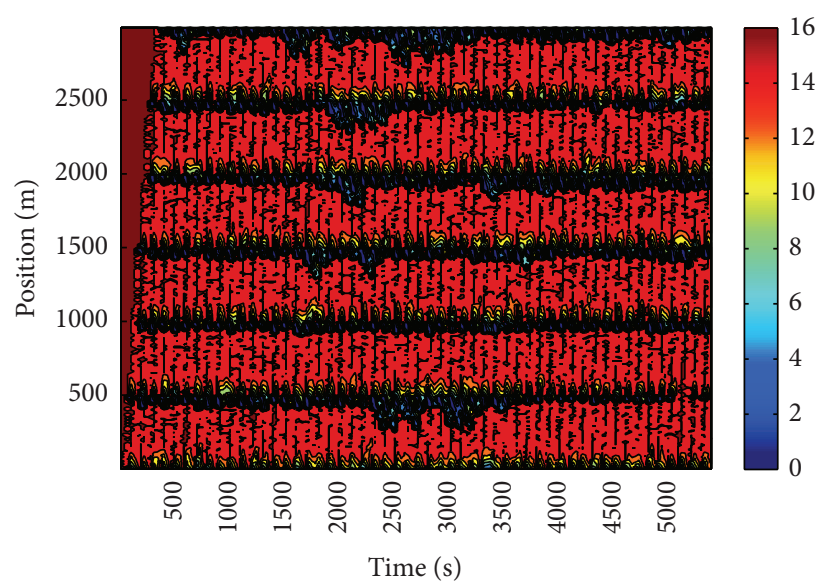

(c) Arrival rate is $1200 \mathrm{vph}$
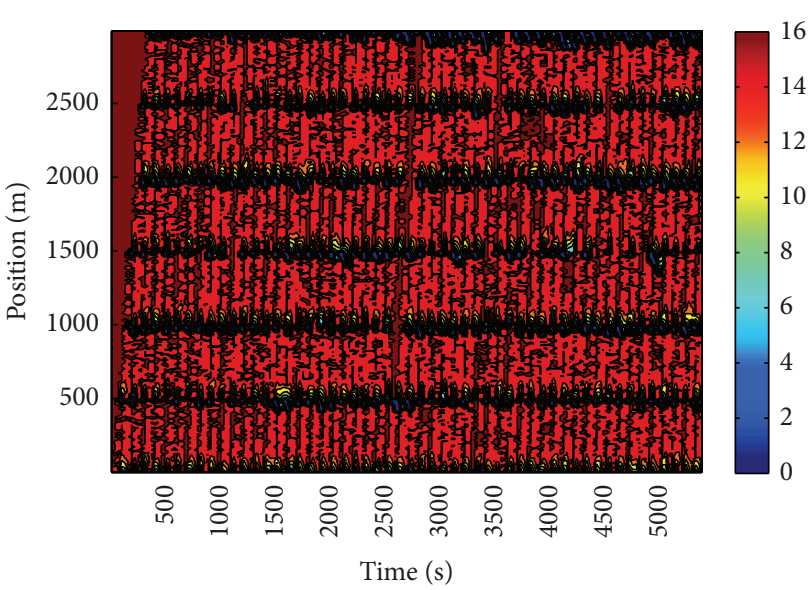

(b) Arrival rate is $800 \mathrm{vph}$

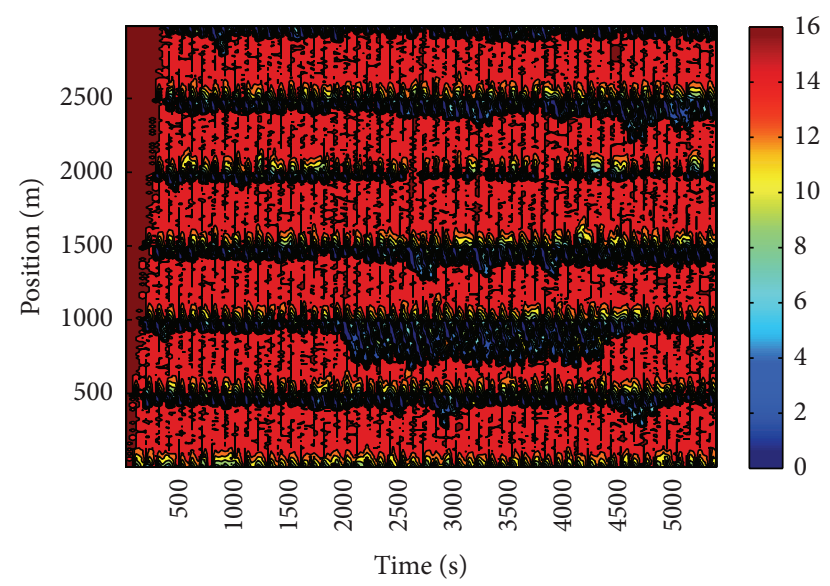

(d) Arrival rate is $1600 \mathrm{vph}$

FIGURE 11: Speed contour plot in vehicle-actuated traffic signals.

TABLE 3: Comparison among different arrival rates.

\begin{tabular}{lcc}
\hline $\begin{array}{l}\text { Arrival rate } \\
\text { (vph) }\end{array}$ & $\begin{array}{c}\text { Number of } \\
\text { sensors }\end{array}$ & Sensor location pattern \\
\hline 400 & & $200,300,500,1100,1400,1800$ \\
800 & 6 & $400,500,1200,1400,1900,2800$ \\
1200 & & $500,600,1900,2200,2600,2900^{*}$ \\
1600 & & $600,900^{*}, 1300,1400,2100,2600$ \\
\hline 400 & 5 & $600,700,1300,1500,2200$ \\
800 & & $100,500,1800,1500,1900$ \\
1200 & & $900^{*}, 1000,1900,2300,3000$ \\
1600 & & $600,1000,1100,1700$ \\
400 & 4 & $400,500,1400,2600$ \\
800 & & $300,700,1300,1900$ \\
1200 & & \\
1600 & &
\end{tabular}

${ }^{*}$ The sensor is located in queuing area.

\section{Conclusion}

The paper studies the sensor location problem in urban arterial street for travel time estimation and proposes optimal

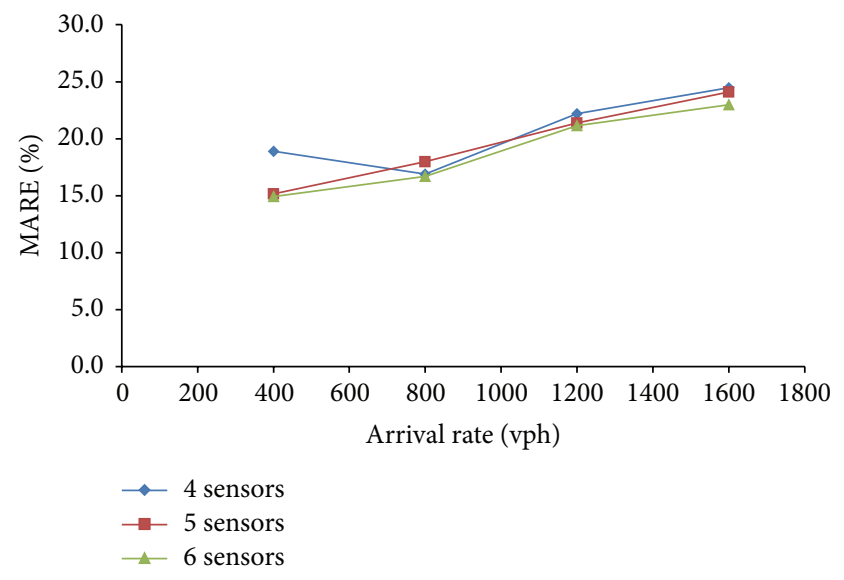

FIgURE 12: Comparison among MARE in different number of sensors for vehicle-actuated traffic signals.

sensor location model (M1) to obtain the minimum travel time estimation error. Based on this model, the influence of traffic signals strategies on sensor location is also discussed. 
By comparing the synchronized traffic signals, green wave traffic signals, and vehicle-actuated signals, it is found that sensor should not be placed in vehicle queuing area. If the sensor is located in the queuing area, its associated coverage area should include the vehicle queuing area as precise as possible. Intersection stop line is an ideal sensor position. There is not any fixed sensor position that can cope with all traffic conditions, and the sensor location should be determined according to the characteristics of traffic flow on the road. Under simple traffic situation where vehicle speed is stable and speed fluctuation is small, few sensors can accurately estimate travel time. In case of complex traffic conditions with large fluctuations of vehicle speed, more sensors are required to estimate travel time.

The future research direction can be considered as follows: (i) our study only takes into account the traffic condition of single lane with fixed traffic volume, and the future research can consider more complex traffic situations, such as the dynamic changes of multilane road with dynamic traffic volume and other real phenomena that match with actual road conditions. (ii) The future research can take this study on urban arterial street as background, taking into account the vehicle turnings, and study the sensor location under the road network layout. (iii) A more efficient model for algorithm should be explored in the future research, although the genetic algorithm used in the paper is an effective solution model. (iv) The study only seeks the optimal sensor location for travel time estimation. Future research can focus on optimized combination of more traffic information applications or more information applications.

\section{Conflict of Interests}

The authors declare that there is no conflict of interests regarding the publication of this paper.

\section{Acknowledgments}

This research was supported by the National Natural Science Foundation Council of China under Projects 71301115, 71431005, 71271150, and 71101102 and Specialized Research Fund for the Doctoral Program of Higher Education of China (SRFDP) under Grant no. 20130032120009.

\section{References}

[1] H. M. Zhang, "Link-journey-speed model for arterial traffic," Transportation Research Record, vol. 1676, no. 1, pp. 109-115, 1999.

[2] A. Skabardonis and N. Geroliminis, "Real-time estimation of travel times on signalized arterials," Transportation and Traffic Theory, no. LUTS-ARTICLE-2009-003, pp. 387-406, 2005.

[3] H. X. Liu and W. Ma, "A virtual vehicle probe model for timedependent travel time estimation on signalized arterials," Transportation Research Part C: Emerging Technologies, vol. 17, no. 1, pp. 11-26, 2009.

[4] S. Takaba, T. Morita, T. Hada, T. Usami, and M. Yamaguchi, "Estimation and measurement of travel time by vehicle detectors and license plate readers," in Proceedings of the Vehicle
Navigation and Information Systems Conference, vol. 2, pp. 257267, IEEE, October 1991.

[5] R. L. Cheu, D. H. Lee, and C. Xie, "An arterial speed estimation model fusing data from stationary and mobile sensors," in Proceedings of the IEEE Intelligent Transportation Systems Proceedings, pp. 573-578, August 2001.

[6] D. J. Dailey and F. W. Cathey, "AVL-equipped vehicles as traffic probe sensors," Tech. Rep. WA-RD 534.1, Washington State Department of Transportation, 2002.

[7] R. Li, G. Rose, and M. Sarvi, "Evaluation of speed-based travel time estimation models," Journal of Transportation Engineering, vol. 132, no. 7, pp. 540-547, 2006.

[8] J. W. C. Van Lint, "Empirical evaluation of new robust travel time estimation algorithms," Transportation Research Record, vol. 2160, no. 1, pp. 50-59, 2010.

[9] D. Ni and H. Wang, "Trajectory reconstruction for travel time estimation," Journal of Intelligent Transportation Systems, vol. 12, no. 3, pp. 113-125, 2008.

[10] H. Yang and J. Zhou, "Optimal traffic counting locations for origin-destination matrix estimation," Transportation Research, Part B: Methodological, vol. 32B, no. 2, pp. 109-126, 1997.

[11] L. Bianco, G. Confessore, and P. Reverberi, "A network based model for traffic sensor location with implications on O/D matrix estimates," Transportation Science, vol. 35, no. 1, pp. 5060, 2001.

[12] M. Gentili and P. B. Mirchandani, "Locating active sensors on traffic networks," Annals of Operations Research, vol. 136, no. 1, pp. 229-257, 2005.

[13] A. Ehlert, M. G. H. Bell, and S. Grosso, "The optimisation of traffic count locations in road networks," Transportation Research Part B: Methodological, vol. 40, no. 6, pp. 460-479, 2006.

[14] X. Fei, H. S. Mahmassani, and S. M. Eisenman, "Sensor coverage and location for real-time traffic prediction in large-scale networks," Transportation Research Record: Journal of the Transportation Research Board, vol. 2039, no. 1, pp. 1-15, 2007.

[15] S. M. Eisenman, X. Fei, X. Zhou, and H. S. Mahmassani, "Number and location of sensors for real-time network traffic estimation and prediction: sensitivity analysis," Transportation Research Record, vol. 1964, no. 1, pp. 253-259, 2006.

[16] E. Castillo, J. M. Menéndez, and P. Jiménez, “Trip matrix and path flow reconstruction and estimation based on plate scanning and link observations," Transportation Research Part B: Methodological, vol. 42, no. 5, pp. 455-481, 2008.

[17] R. Mínguez, S. Sánchez-Cambronero, E. Castillo, and P. Jiménez, "Optimal traffic plate scanning location for OD trip matrix and route estimation in road networks," Transportation Research Part B: Methodological, vol. 44, no. 2, pp. 282-298, 2010.

[18] S.-R. Hu, S. Peeta, and C.-H. Chu, "Identification of vehicle sensor locations for link-based network traffic applications," Transportation Research Part B: Methodological, vol. 43, no. 89, pp. 873-894, 2009.

[19] F. Viti, M. Rinaldi, F. Corman, and C. M. Tampère, "Assessing partial observability in network sensor location problems," Transportation Research Part B: Methodological, vol. 70, pp. 6589, 2014.

[20] N. Zhu, Y. Liu, S. Ma, and Z. He, "Mobile traffic sensor routing in dynamic transportation systems," IEEE Transactions on Intelligent Transportation Systems Magazine, vol. 15, no. 5, pp. 22732285, 2014. 
[21] H. X. Liu and A. Danczyk, "Optimal sensor locations for freeway bottleneck identification," Computer-Aided Civil and Infrastructure Engineering, vol. 24, no. 8, pp. 535-550, 2009.

[22] Y. Liu, N. Zhu, S. Ma, and N. Jia, "Traffic sensor location approach for flow inference," IET Intelligent Transport Systems, vol. 9, no. 2, pp. 184-192, 2015.

[23] J. Kim, B. Park, J. Lee, and J. Won, "Determining optimal sensor locations in freeway using genetic algorithm-based optimization," Engineering Applications of Artificial Intelligence, vol. 24, no. 2, pp. 318-324, 2011.

[24] J. Kianfar and P. Edara, "Optimizing freeway traffic sensor locations by clustering global-positioning-system-derived speed patterns," IEEE Transactions on Intelligent Transportation Systems, vol. 11, no. 3, pp. 738-747, 2010.

[25] J. Kwon, K. Petty, and P. Varaiya, "Probe vehicle runs or loop detectors? Effect of detector spacing and sample size on accuracy of freeway congestion monitoring," Transportation Research Record, vol. 2012, pp. 57-63, 2007.

[26] G. B. Thomas, "The relationship between detector location and travel characteristics on arterial streets," ITE Journal, vol. 69, no. 10, pp. 36-42, 1999.

[27] X. Ban, L. Chu, R. Herring, and J. D. Margulici, "Sequential modeling framework for optimal sensor placement for multiple intelligent transportation system applications," Journal of Transportation Engineering, vol. 137, no. 2, pp. 112-120, 2010.

[28] J. W. C. van Lint, "Online learning solutions for freeway travel time prediction," IEEE Transactions on Intelligent Transportation Systems, vol. 9, no. 1, pp. 38-47, 2008.

[29] L. D. Vanajakshi, B. M. Williams, and L. R. Rilett, "Improved flow-based travel time estimation method from point detector data for freeways," Journal of Transportation Engineering, vol. 135, no. 1, pp. 26-36, 2009.

[30] L. Li, X. Chen, Z. Li, and L. Zhang, "Freeway travel-time estimation based on temporal-spatial queueing model," IEEE Transactions on Intelligent Transportation Systems, vol. 14, no. 3, pp. 1536-1541, 2013.

[31] J. T. Morgan and J. D. C. Little, "Synchronizing traffic signals for maximal bandwidth," Operations Research, vol. 12, no. 6, pp. 896-912, 1964.

[32] R. Hall, Handbook of Transportation Science, Springer, Berlin, Germany, 1999. 


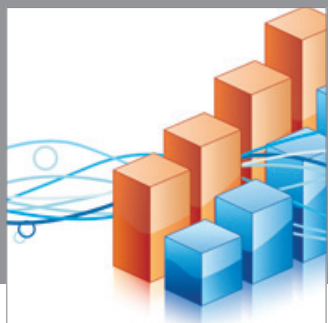

Advances in

Operations Research

mansans

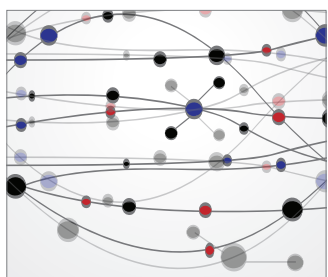

The Scientific World Journal
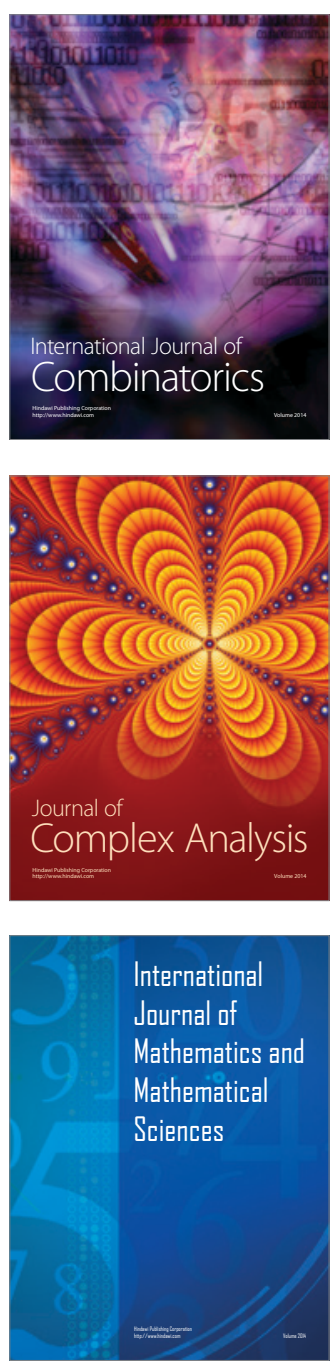
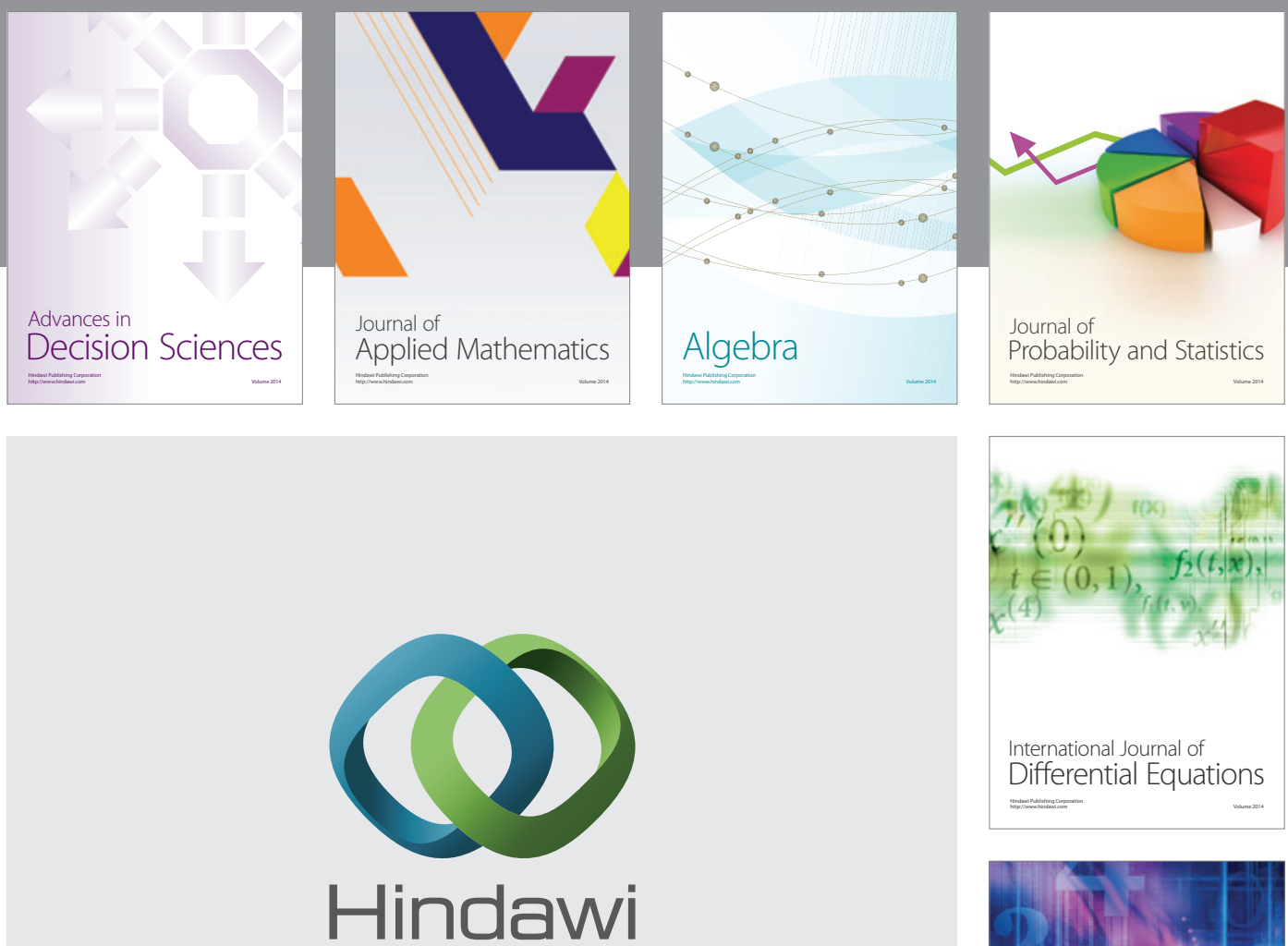

Submit your manuscripts at http://www.hindawi.com
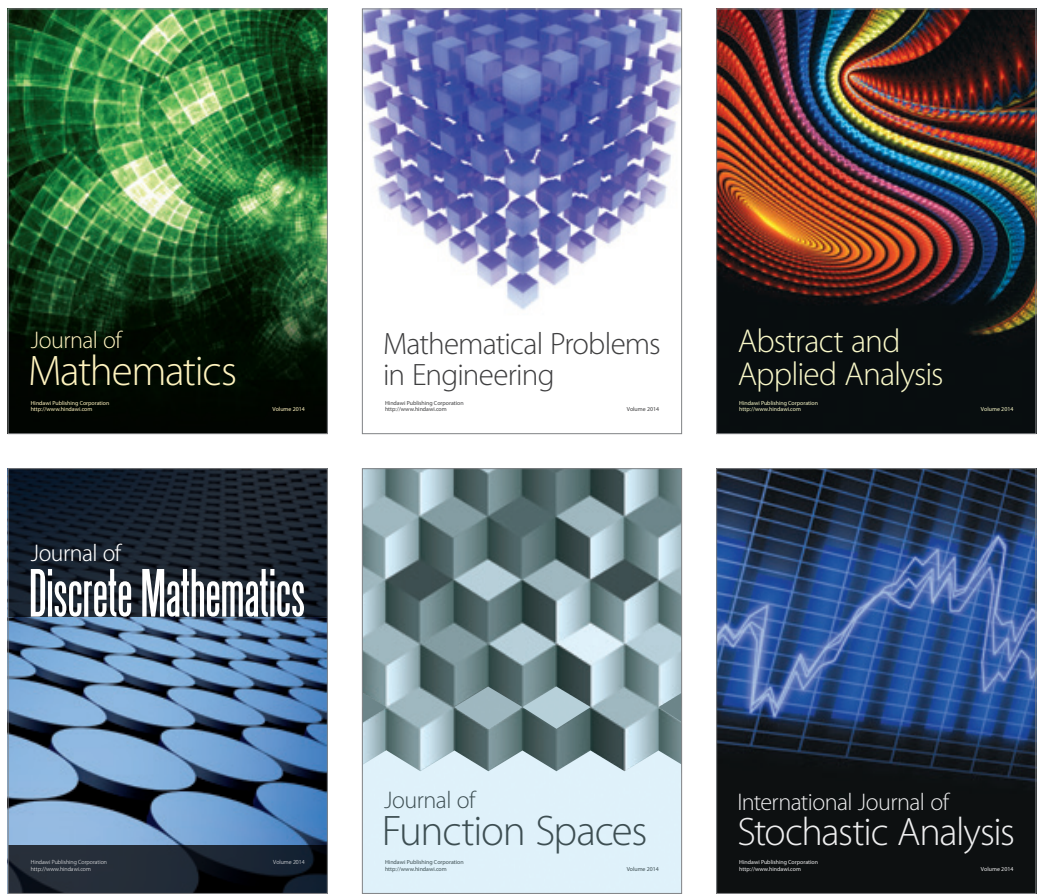

Journal of

Function Spaces

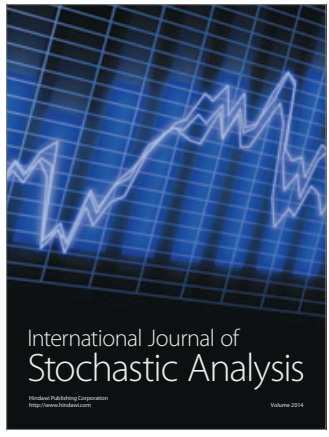

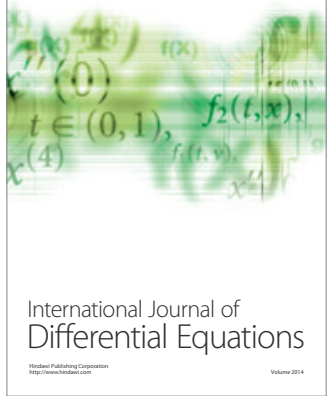
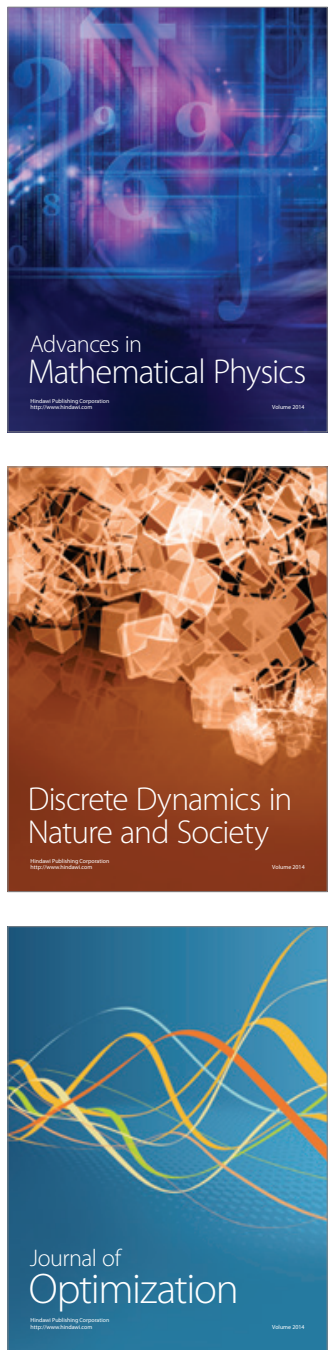